\title{
Original Article \\ Simple Software Solution for Portfolio Management for Medical Consultants
}

\author{
Lakshan M.T.D. \\ District General Hospital, Kuliyapitiya, Sri Lanka.
}

\section{Introduction:}

Portfolio management is a standard professional requirement and, in certain health care delivery systems, a mandatory prerequisite for licensing and revalidation 1.

\section{Ideal Portfolio System}

An ideal medical portfolio system should have following characteristics:

1 Simple to use

2. Readily accessible - i.e. portable

3. Able to document key areas of professional life according to defined outcome framework

4. Should store data in a confidential manner

5. A non-technical person should be able to fill in the details, especially when data entry is delegated.

6. Photo and video documentation should be possible - particularly useful for specialities like surgery.

7. Ability to assess the content by authorized person (assessor) for purposes like Continuous Professional Development (CPD) point allocations and revalidation

8. Should not pose an additional burden to already busy schedule of clinicians

While it is undisputed that portfolio maintenance plays a pivotal role in legitimate professional conduct, "how" and "what to collect" is not universally agreed upon.

\section{Areas for data collection}

GMC has published the areas for data collection in the Guide to Good Medical Practice.2

Key domains mentioned are:

1. Knowledge, skills and performance

2. Safety and Quality

3. Communication, partnership and teamwork

4. Maintaining trust

Data collection points of a portfolio can be made to represent above domains.

\section{Portfolio methodology}

There are many methods for maintaining a portfolio. Traditionally, the "pen and paper" method has been used. With the advent and ubiquitous use of information technology and World Wide Web, there is an increasing trend towards using software for maintaining a digital portfolio.

\section{Available Systems}

While there are web based sophisticated portfolio systems available particularly via Royal Colleges of the UK, when applying to different health care systems there are problems such as:

1. They are tailor-made for specific health care systems e.g., NHS of UK

2. They have specific aims e.g. revalidation, appraisal, Professional Development Plan (PDP) built in to those. By the very nature of this allinclusive approach, the system tends to be very sophisticated and complex. 
ISSN : $2012-855 x$

Ceylon Journal of Otolaryngology

(C) 2016; College of Otorhinolaryngologists and Head and Neck Surgeons of Sri Lanka

3. They are bound to tight schedules based on deadlines.

4. Usually there is a payment to be made either individually or by the professional body.

\section{Methodology}

Using open source platform Drupal - a PHP based content management system, a simple yet robust portfolio system has been developed.

9 data collection points aiming at four domains have been identified.

1. Academic qualifications

2. Employment details

3. Logbook entry

4. Research and publications

5. Reflections in to practice

6. Teaching and Training

7. Workshops, Seminars and Meetings

8. Professional Commitments

9. Social Services

The software has been developed to be able to collect data pertaining to above 9 categories. Data entry is done in a simple form and it is made in mobile device friendly format. Videos and Images can be uploaded as file attachment or directly from a camera of a mobile phone. Files in the common formats like Microsoft PowerPoint, Word, Excel, JPG and PDF can be uploaded as well.

Collected data can be retrieved as and when needed and the output can be downloaded in common office document formats of Microsoft Word and Microsoft Excel.

These files can be further edited / formatted before submission for any form of assessment.

\section{Screenshots}

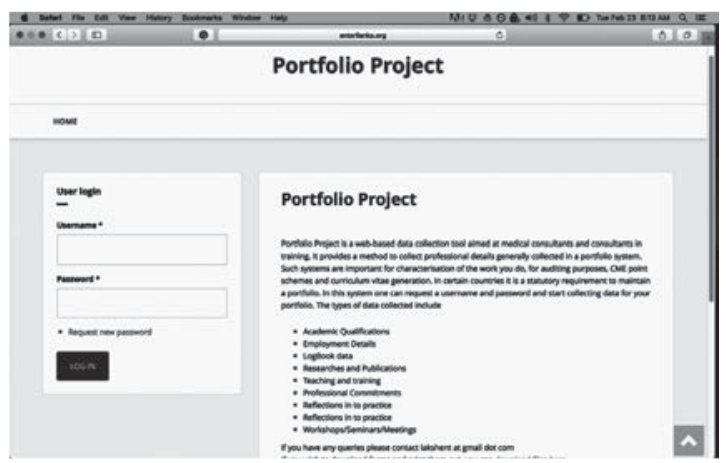

Fig 1 - Welcome Page

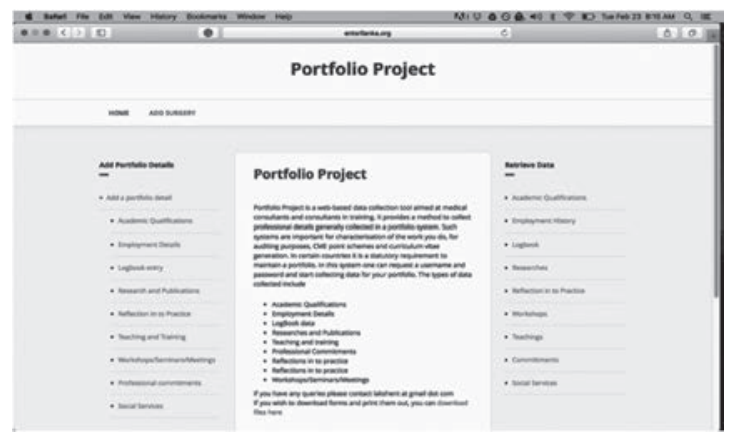

Fig 2 - Logged in Page Screenshots of the pages for data collection

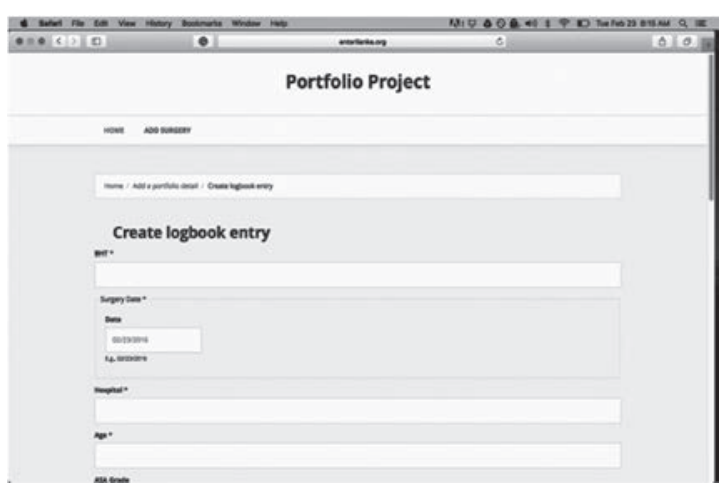

Fig 3 - Logbook entry form

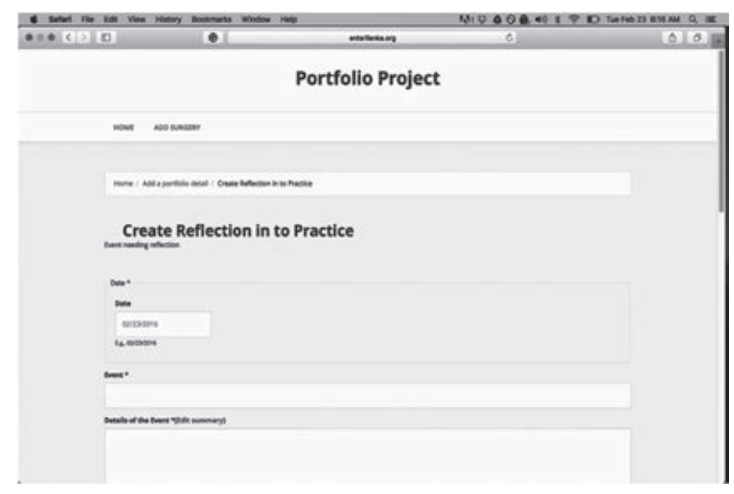

Fig 4 - Reflection in to practice form 
A demo account is created and should the reader wish the system can be tested by logging with the user name demo and password demo.

http://www.entsrilannka.org/portfolio

\section{Following are highlights of this software:}

1. User can enter portfolio details in above mentioned data points via a simple interface

2. User can retrieve data in common office document formats (MS DOC, EXCEL)

3. Further modification can be done and printouts can be obtained

4. Multimedia of surgeries like images and videos can be uploaded

5. Mobile phone can be used to enter data

6. Universally available whenever the user needs it

7. Assessment ready

8. Its scalable - can be increased to any number of user accounts based on server capacity and specifications

9. Data security is tightly maintained

\section{Current Usage}

After endorsement by the College of Otorhinolaryngologists and Head and Neck surgeons of Srilanka, its membership will get full access to it. General surgeons and trainees from both Srilanka and abroad on personal invitations are also using the system. A free account can be requested by emailing lakshent@gmail.com

\section{Future}

Further software development is planned to create mobile apps to access the system.

Off line data entry is another highly requested feature.

Data collection on usage patterns will be performed.

Further endorsements by professional bodies will result in expansion of the user base and based on periodic feedback further improvements will be made.

\section{References:}

1. http://www.gmc-uk.org/doctors/revalidation/ revalidation_information.asp

2. Good Medical Practice - framework for appraisal - General Medical Council - UK http://www.gmcuk.org/GMP_framework_for_ appraisal_and_revalidation.pdf

3. Portfolio System: http://www.entsrilanka.org/ portfolio 